\title{
Athlete Rehabilitation Evaluation System Based on Internet of Health Things and Human Gait Analysis Algorithm
}

\author{
Chen Lianzhen $\mathbb{I D}^{1}$ and Zhu Hua \\ ${ }^{1}$ Lingnan Normal University, Zhanjiang, Guangdong 524048, China \\ ${ }^{2}$ Guangdong Medical University, Zhanjiang, Guangdong 524023, China \\ Correspondence should be addressed to Chen Lianzhen; 160407101@stu.cuz.edu.cn
}

Received 3 December 2020; Revised 6 January 2021; Accepted 1 April 2021; Published 3 September 2021

Academic Editor: Wei Wang

Copyright (c) 2021 Chen Lianzhen and Zhu Hua. This is an open access article distributed under the Creative Commons Attribution License, which permits unrestricted use, distribution, and reproduction in any medium, provided the original work is properly cited.

\begin{abstract}
In order to improve the effect of athlete's injury recognition and rehabilitation evaluation, this paper studies the traditional rehabilitation evaluation method and proposes a new athlete rehabilitation evaluation system combining the Internet of Health Things technology and human gait analysis algorithm. Moreover, this paper combines sports characteristics to improve the algorithm of human gait analysis. In addition, through the study of the athlete's human body modeling and movement process, a human gait analysis algorithm that can be applied to multiple sports is proposed, and the gait parameter analysis and algorithm reliability research are carried out through simulation analysis. After confirming that the algorithm is effective, this paper combines the Internet of Health Things technology to construct a system model, obtains the system function module architecture with the support of the Internet of Health Things technology, and conducts experiments to verify the system performance. From the experimental research, it can be seen that the model constructed in this paper meets the theoretical and practical needs, and the system in this paper can be applied to practice in the future. The human gait recognition algorithm constructed in this article has a good effect and can play an important role in sports rehabilitation of athletes. At the same time, the system constructed in this article has certain advantages over traditional sports rehabilitation systems with the support of algorithms.
\end{abstract}

\section{Introduction}

Sports injury is an important problem in the development of competitive sports. With the continuous improvement of the level of competitive sports in my country, people's awareness of sports injuries is also increasing, and the research on sports injuries is also increasing. Competitive sports is the most widely valued in my country's sports industry, and sports injury is also a problem to be solved that hinders the development of competitive sports in my country.

Sports are a collective sport that uses techniques such as running, jumping, shooting, and physical confrontation. As sports are interspersed with various complex and changeable techniques and tactics and fierce physical confrontations, sports are extremely variable and enjoyable. Due to the many characteristics of sports events, sports injuries are frequent.
In foreign studies, some scholars have suggested that sports are not a high-risk sport in a sense. However, even within the limits of the rules, reasonable physical contact and actions beyond the normal capacity of the human body will still occur during the training process. In particular, as the intensity of the confrontation increases, the probability of more and more injuries from physical contact also increases [1]. The development of modern sports is gradually developing towards higher intensity, faster frequency, and more intense high-altitude confrontation [2]. While sports quickly attract the public's attention, it also significantly increases the incidence of sports injuries in sports. Moreover, modern sports require relatively high physical fitness, and correct functional movements will provide support for athletes' excellent physical fitness and improve sports performance. In sports confrontation, athletes often cannot control the correct movements, which leads to sports injuries. 
The application of gait analysis technology and equipment to solve clinical problems in the fields of orthopedics and sports rehabilitation has developed rapidly. The patient cannot return to a healthy state immediately after the operation of the lower limbs, and it also requires postoperative scientific observation and sports training. At the same time, the doctor can also modify and improve the sports rehabilitation treatment plan in time according to the patient's postoperative rehabilitation [3].

The contributions of this paper are as follows: (1) combining Internet of Things technology and human gait analysis algorithm to propose a new athlete rehabilitation evaluation system; (2) improving the human gait analysis algorithm based on sports characteristics, and proposing a human gait analysis algorithm that can be applied to multiple sports through the athlete's human body modeling and movement process; (3) optimizing DMPs and establishing the mapping relationship between step height and weight based on multiple curves of the same type and different heights, and then the height of the stairs can directly affect the gait style and avoid the unbalance of the curve scaling.

In order to accurately measure gait parameters, gait analysis equipment that objectively evaluates the effect of treatment has also emerged. Therefore, the combination of Internet of Health Things technology and human gait analysis algorithm can effectively improve the rehabilitation evaluation effect of athletes, facilitate the formulation of effective rehabilitation plans in time, and improve the rehabilitation effect of rehabilitation athletes and the recovery of sports ability after injury.

\section{Related Work}

At present, the research on human gait mainly uses motion images and plantar pressure for data analysis. The kinematics data of human gait analysis mainly use high-speed cameras to capture motion images. The coordinates of key points are used to obtain key data such as the speed of key parts, the change of the height of the center of gravity, and the angle change of the main joint points. The literature [4] summarized the accelerometer measurement system used based on different measurement tasks and used a three-axis accelerometer to measure a moving human body. The literature [5] evaluated and verified the effectiveness of the integrated accelerometer to measure dynamic acceleration by comparing the measurement results of the goniometer and the integrated accelerometer. In order to obtain more human kinematics data, a combination of sensors such as gyroscopes, magnetoresistive sensors, and accelerometers can also be used in the measurement and research of human gait. The angle changes of key parts such as the ankle joint and knee joint can be obtained by installing on the foot, calf, thigh, and other parts. In addition, flexible goniometers, ETS, and sensing fabrics can also be used as wearable sensors for human gait analysis. The use of flexible goniometers to measure joint angles has been used in clinical research [6]. The accuracy of ETS's acquisition of target location and orientation data is similar to that of image analysis [7], but the disadvantage is that it is susceptible to magnetic interference from metal objects within the measurement range. As an ideal portable sensor, smart sensing fabrics have been used by researchers to conduct gait research by using socks with sensors [8]. From the perspective of future development trends, portable mobile sensors will be the main development direction of future kinematic data collection. However, the main problems faced by the current portable motion sensors are the calculation of the initial value and the accumulation of errors. Therefore, at present, scientists are completing future gait testing and analysis tasks by developing newer sensors and test methods. The main measurement data of dynamics are the force between man and the ground. When the human body is doing actions such as walking, running, and jumping, it will generate pressure on the ground. At the same time, it will also receive an equal and opposite reaction force from the ground. This force is the plantar pressure. The force on the sole of a healthy adult is mainly concentrated in the second and third metatarsals and heels [9]. The distribution characteristics of plantar pressure have become one of the important bases for medical diagnosis and technical action diagnosis.

This article mainly studies the application of Internet of Health Things in the rehabilitation of athletes. Some experts and scholars have done similar research before. The literature [10] proposed a 3D human joint action and gait recognition method. This research uses an unlabeled posture recovery method to extract movement features and structural features. The two types of features extracted are used to automatically extract 3D human joint information for action and gait recognition. The literature [11] proposed a $3 \mathrm{D}$ gait recognition method. This work reconstructs a $3 \mathrm{D}$ human body model from video sequences taken by multiple cameras to generate two feature sets. These two feature sets include the static feature set for the length of the key segment and the dynamic feature set for identifying the movement trajectory of the lower limbs. Moreover, this method combines static and dynamic feature sets to achieve a recognition accuracy of $70 \%$ and can well solve the impact of view and surface changes. In order to ensure the accuracy of 3D motion tracking, the literature [12] proposed a 3D unmarked motion tracking algorithm for obtaining human gait recognition motion data. The algorithm uses a marker-based motion capture system to generate ground truth data to evaluate the unmarked motion tracking system. At the same time, this method selects three classifiers: naive Bayes, multilayer perceptron, and support vector machine for recognition and identification. Compared with the other two classifiers, the support vector machine classifier achieves an accuracy of $93.5 \%$. In order to provide rich $3 \mathrm{D}$ gait data, the literature [13] used the second-generation KinectV2 tool to create a 3D skeleton-based gait database. The data set contains $3 \mathrm{D}$ information of joints and corresponding 2D contour images, and static and dynamic features can be extracted from it for gait recognition. Moreover, under different viewing angle changes, the classification rate on the database can reach more than $90 \%$. The literature [14] proposed a new method of gait recognition based on the fusion of dynamic and static features. This method selects the gait energy map as the static 
feature, stride length, and step frequency as the dynamic feature and performs feature fusion. At the same time, the literature proposed a kernel principal component analysis method combined with local preserving projection to reduce the gait feature size (KPCA-LPP).

The literature [15] proposed a gait recognition method that calculates standardized views of pedestrian body parts trajectories. This method first calculates the walking view during half of the gait cycle, then applies the homography transformation to approximately transform the walking view to the parallel view, and finally applies the homography to the trajectory of the head and feet during the half gait cycle. The literature [16] used enhanced human contours to remove artifacts and obtain the width and height of the main body, automatically detect body joints, determine the joint angle trajectory, and determine the crotch height and step length of the walking object. Moreover, it obtains the gait feature with the same viewing angle through perspective transformation. The experimental results show that the correct classification rate of this method is above $90 \%$. The literature [17] used the method of gait self-calibration to perform gait recognition. This method does not need to know the internal or external camera parameters and will not attract the attention of the recognized person. The results of the experiment show that the use of pure dynamic gait features in all views can achieve a classification rate of $73.6 \%$.

The literature [18] proposed a gait recognition method using frequency domain features and view transformation model (VTM). This method mainly uses the Fourier analysis of the gait cycle to extract the frequency domain features from the space-time contour volume of the pedestrian and construct the VTM. The VTM is used to convert the gait features into the same perspective and perform similarity measurement. The literature [19] proposed a VTM based on optimized gait energy (GEI). This method first constructs the spatial domain GEI from the complete walking period, then optimizes the constructed GEI by linear discriminant analysis, and finally creates the VTM by applying truncated singular value decomposition on the optimized GEI. The literature [20] developed a Gaussian process classification framework to estimate the perspective of each detected gait sequence, used canonical correlation analysis to simulate the correlation of gait sequences from different views, and used the correlation strength as a similarity metric. This method is more robust to noise. The literature [21] proposed a gait recognition method based on view-invariant discriminant projection, which mainly uses iterative learning to find the best single linear projection to improve the discrimination ability of multiview gait features. Moreover, because it relies on the view invariance to judge the projection, it can directly match the multiview gait features without knowing or estimating the viewing angle information. The literature [22] proposed a discriminant projection with list constraints (DPLC) to deal with the view variance in cross-view gait recognition and automatically capture the main discriminative information by introducing correction terms. At the same time, a simple and effective gait representation based on gait energy images is introduced. That is, it uses the gait personality image (GII) to better capture the discrimination information for cross-gait recognition.

The abovementioned related research not only analyzes the existing and faced problems in the management of sports rehabilitation in my country from the theoretical level but also provides many practical and effective measures to realize the orderly progress of the rehabilitation of persons with disabilities from the specific path of practice and future development. Therefore, this paper studies the traditional rehabilitation evaluation methods and proposes a new athlete rehabilitation evaluation system combining the Internet of Things technology and human gait analysis algorithms. Combining sports characteristics to improve the human body gait analysis algorithm, through the athlete's body modeling and movement process, a human body gait analysis algorithm that can be applied to multiple sports is proposed, and the gait parameter analysis and algorithm reliability are carried out through simulation analysis.

\section{Dynamic Motion Primitive Algorithm Based on the Coupling of Sole Pressure}

As the basic algorithm of Internet of Health Things through gait recognition, the core idea of the dynamic primitive algorithm is to update the weight parameters of the relevant forcing items through the local weighting algorithm and to adjust the gait curve through the target point by changing the target of the point attraction Internet of Health Things system. In specific use, the target curve is used as a teaching curve. Through learning the target curve, it is modulated according to the target point, and the generated curve can well retain its own characteristics. In this paper, DMPs are used to learn the curve of athletes' sports rehabilitation, and then the curve is dynamically modulated by target points to achieve different degrees of rehabilitation. The DMP algorithm comes from the spring damper model, which is a second-order dynamic Internet of Health Things system. When an external force is applied, it can produce more complex trajectories. The spring damping term can be expressed as [23]

$$
y^{\prime \prime}=\alpha_{y}\left(\beta_{y}(g-y)-y^{\prime}\right) .
$$

Among them, $\alpha_{y}$ is the gain term of the Internet of Health Things system, $\beta_{y}$ is a constant term, $g$ is the set target point, $y$ is the current Internet of Health Things system state, $y^{\prime}$ is the speed of the Internet of Health Things system, and $y^{\prime \prime}$ is the acceleration of the Internet of Health Things system. If there is no external force, the spring damping term always converges to the target $g$, which is like the spring always converges to the 0 position. DMP introduces a forced term $f$ on the basis of the spring damping term. Through the action of the forced term, the Internet of Health Things system can produce more complex behaviors. This is similar to applying a force on the basis of the spring damping term, which will cause the spring to produce a more complex movement. DMP can be expressed at this time as [24] 


$$
y^{\prime \prime}=\alpha_{y}\left(\beta_{y}(g-y)-y^{\prime}\right)+f .
$$

The first-order form of the above formula is

$$
\begin{aligned}
z^{\prime} & =\alpha_{y}\left(\beta_{y}(g-y)-z\right)+f, \\
\tau y^{\prime} & =z .
\end{aligned}
$$

In the above formula, $\tau$ is the time constant. $\alpha_{y}$ and $\beta_{y}$ are constant terms. If the $f=0$ Internet of Health Things system is a stable second-order Internet of Health Things system, it is similar to a spring damper without external force. Moreover, $(z, y)=(0, g)$ is the only point attractor Internet of Health Things system. When the values of $\alpha_{y}$ and $\beta_{y}$ are

$$
\alpha_{y}=\beta_{y} * 4,
$$

the entire Internet of Health Things system will show a critically damped state. $y$ is the output of the Internet of Health Things system at this time. In order to have a better learning curve in DMP, it is necessary to control the more complex $f$, and its complex behavior comes from the control of the virtual force. In order to achieve more complex force control, the virtual force is expressed as a weighted combination of nonlinear radial basis functions in DMP. Of course, it can also be expressed using a linear function. $f$ is defined as

$$
f(t)=\frac{\sum_{i=1}^{N} \psi_{i}(t) \omega_{i}}{\sum_{i=1}^{N} \psi_{i}(t)}
$$

Among them, $\psi_{i}(t)$ is a fixed basis function, and $\omega_{i}$ is an adjustable weight. $i$ is the order of the weights, and the above formula realizes the nonlinear expression of the forcing term. Among them,

$$
\psi_{i}=\exp \left(-h_{i}\left(x-c_{i}\right)^{2}\right),
$$

is a Gaussian kernel function with $c_{i}$ as the center and $h_{i}$ as the variance. It can be understood as the result of weighting and normalizing multiple virtual forces with Gaussian functions. Moreover, it is also equivalent to performing Gaussian coding on this behavior [25]. Due to the time dependency between the Internet of Health Things systems, it is not allowed to directly couple with other dynamic Internet of Health Things systems and cannot be decoupled, which will cause the Internet of Health Things system to be inflexible and changeable. Therefore, a new method is proposed to replace the time term with a first-order linear follow method as a standard Internet of Health Things system, as shown in the following formula:

$$
x^{\prime}=-\alpha_{x} x,
$$

where $\alpha_{x}$ is a constant term. At the beginning, an initial state $x_{0}$ is chosen arbitrarily, such as $x_{0}=1 . x$ can be used as a phase variable, understood as a time term, and used to control execution. However, unlike the time item, each Internet of Health Things system is independent. When $x$ monotonously converges to 0 , it means that the target $g$ has been reached. At this time, $x=0$ is a stable point in the Internet of Health Things system. The use of the standard Internet of Health Things system is as follows, and we define $x_{0}=1$. When $x$ decays to 0 , the Internet of Health Things system tends to be stable. As shown in Figure 1, the vertical axis is the value of $x$, and the horizontal axis is time [26].

After the standard items are determined, they need to be introduced into the DMP, and the period defined by the point attraction Internet of Health Things system is given. Later, the standard Internet of Health Things system is introduced into the second-order Internet of Health Things system:

$$
f(x, g)=\frac{\sum_{i=1}^{N} \psi_{i}(t) \omega_{i}}{\sum_{i=1}^{N} \psi_{i}} x\left(g-y_{0}\right) .
$$

Among them, $y_{0}$ represents an initial position of $y, g$ is the target point, and the modulation of $x$ means that the forced term will disappear when it reaches the target $g$, which ensures the stability of the Internet of Health Things system. It can be found that $\psi_{i}$ has nothing to do with the time term, and the specific control is related to $x$; that is, $x$ controls the output of the Gaussian kernel function. The modulation of $g-y_{0}$ affects the amplitude of the model and has spatial scaling characteristics. In general, $g$ and $y_{0}$ are not equal. If they are equal, DMP will fail. The specific reason is that $g-y_{0}=0$ will cause the overall result of the above formula to be 0 , which means that the Internet of Health Things system cannot be controlled at this time [27].

The overall block diagram is shown in Figure 2, which includes the standard Internet of Health Things system for controlling the start and end, nonlinear function fitting for the behavior of the control Internet of Health Things system, the conversion Internet of Health Things system for output Internet of Health Things system acceleration, and the coupling term for Internet of Health Things system expansion [28].

From the above analysis, it can be seen that DMP has dynamic modulation of the target point $g$, and the control of the virtual force is affected by the Gaussian kernel function and its weight. This is very convenient for the gait generation of lower limb exoskeleton exercise rehabilitation. Because the process of sports rehabilitation needs to use target points to control the style and amplitude of the curve, the weight can affect the style of the curve.

In order to obtain the weights in DMP, it is necessary to introduce the source of the Gaussian kernel function [29]:

$$
h_{i}=\frac{B F s}{c_{i} \dot{x}}
$$

BFs is the number of Gaussian kernels artificially set according to the required accuracy. $c_{i}$ is the center position of the Gaussian nucleus after $0-1$ equally divided according to the number of Gaussian nuclei. In this way, a complete Gaussian kernel function can be obtained. In order to 


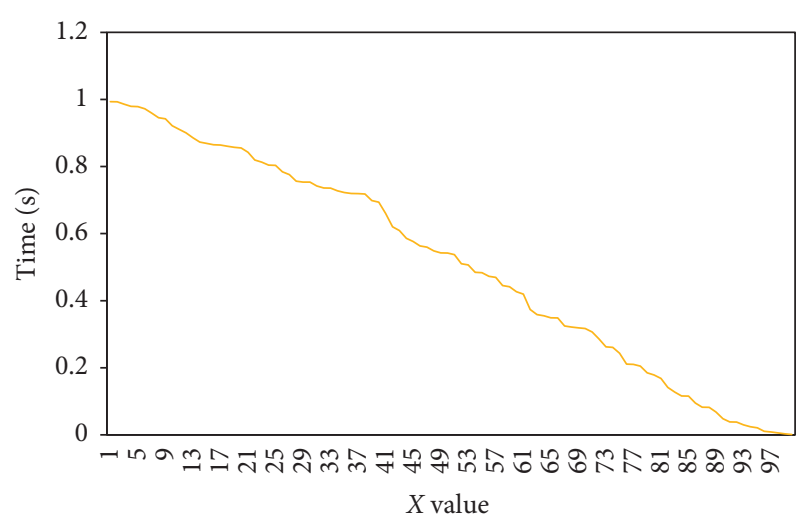

Figure 1: Standard Internet of Health Things system attenuation term $x$.

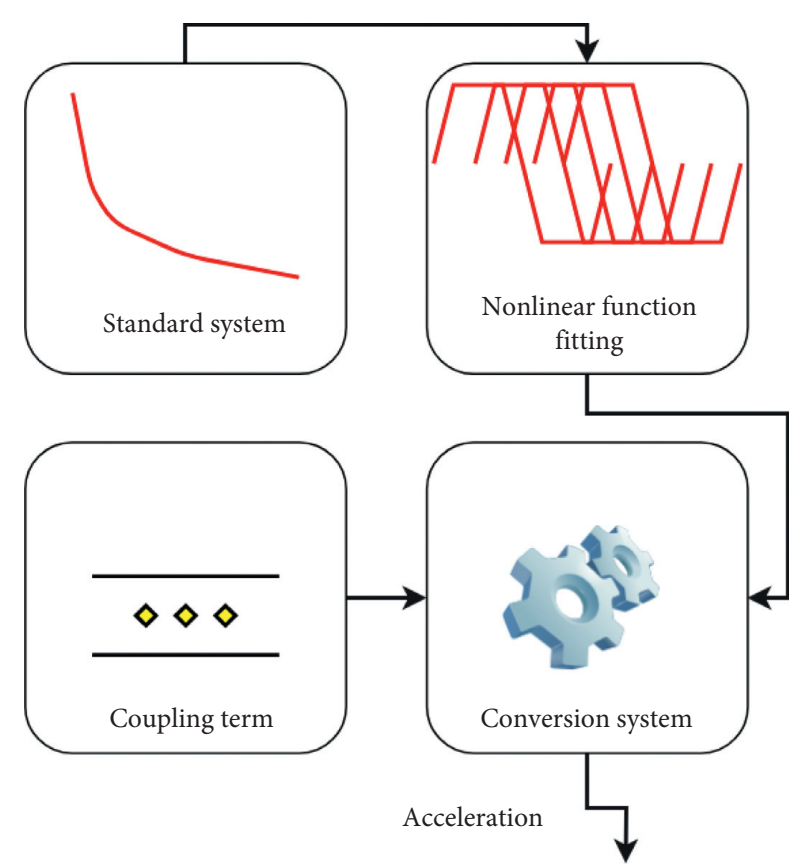

FIgURE 2: Block diagram of the DMP Internet of Health Things system.

obtain the fitting weight of the forced term $f$ in DMP, the virtual force needs to be calculated. The virtual force is calculated:

$$
f_{i}=y_{i}^{\prime \prime}-\alpha_{y}\left(\beta_{y}\left(g-y_{i}\right)-y_{i}^{\prime}\right) .
$$

The acceleration and velocity in the above formula are obtained by difference of the curve. After obtaining $f_{i}$, the weight is obtained through partial weighted regression. The weight expression corresponding to each Gaussian kernel function solved by the local weighted regression algorithm is as follows:

$$
\omega_{i}=\frac{s^{T} \psi_{i} f_{i}}{s^{T} \psi_{i} s}
$$

In the above formula [30],

$$
\begin{aligned}
s & =\left(\begin{array}{c}
x_{t 0}\left(g-y_{0}\right) \\
\vdots \\
x_{t N}\left(g-y_{0}\right)
\end{array}\right), \\
\psi_{i} & =\left(\begin{array}{ccc}
\psi_{i}\left(t_{0}\right) & \cdots & 0 \\
0 & \ddots & 0 \\
0 & \cdots & \psi_{i}\left(t_{N}\right)
\end{array}\right) .
\end{aligned}
$$

Through the above algorithm research, the algorithm can be combined in the research in the following part of the paper, and the algorithm can be used as the basic algorithm for constructing the rehabilitation model to calibrate the sports characteristics of the athletes, so as to facilitate the timely diagnosis of sports rehabilitation.

\section{Gait Planning Based on Dynamic Motion Primitives}

When using DMPs to plan gait, at least one movement successive gait is required as a reference curve input. Then, the weight of DMPs is calculated, the weight is substituted, and the target is set to obtain the acceleration, and then the acceleration can be integrated to obtain the output modulation curve.

The following is the scene of the rehabilitation exercise of the lower extremity exoskeleton. If it is assumed that the scene of exoskeleton movement is limited to the $X$ and $Z$ planes, then the gait curve in this scene will be a two-dimensional curve. For multidimensional curves, the application under the DMP framework is generally to decompose it into singledimensional curves.

For multidimensional Internet of Health Things systems, multidimensional Internet of Health Things systems are generally treated as single-dimensional. The method of using DMP for multidimensionality is represented by DMPs. The influence of the number of different Gaussian kernel functions on the planning curve of DMPs is discussed below. The selected reference curve is the curve of the first step. It can be seen from Figure 3 that as the number of kernel functions increases, the curve accuracy of DMPs planning becomes higher. As shown in Figure 4, the horizontal axis represents the position of the ankle joint from the origin in the horizontal direction, and the vertical axis represents the distance of the ankle joint from the origin in the $Z$-axis direction.

The operating speed of the actual Internet of Health Things system needs to be considered, the operating time of the embedded Internet of Health Things system needs to be compared, and the appropriate Internet of Health Things system parameters are selected. The hardware platform is BeagleBone Black AM3358 CortexA8, the Internet of Health Things system platform is embedded Ubuntu, and the operating speed is $1 \mathrm{GHz}$. The running time comparison of DMPs with different numbers of kernel functions is shown in Table 1 [31]:

It can be known from Table 1 that as the number of Gaussian kernels increases, the running time of the Internet of Health Things system will increase. Therefore, the number 


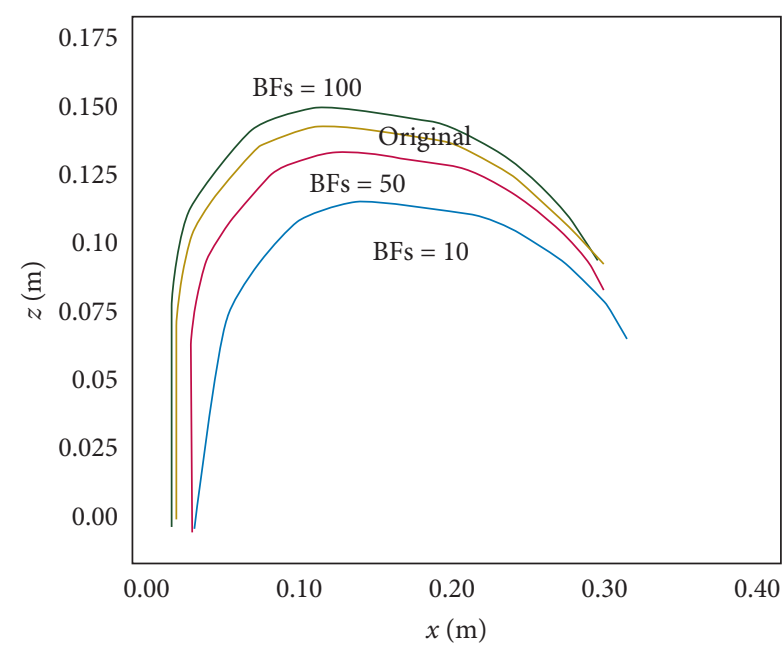

FIgURE 3: The influence of the number of DMPs weight on the curve.

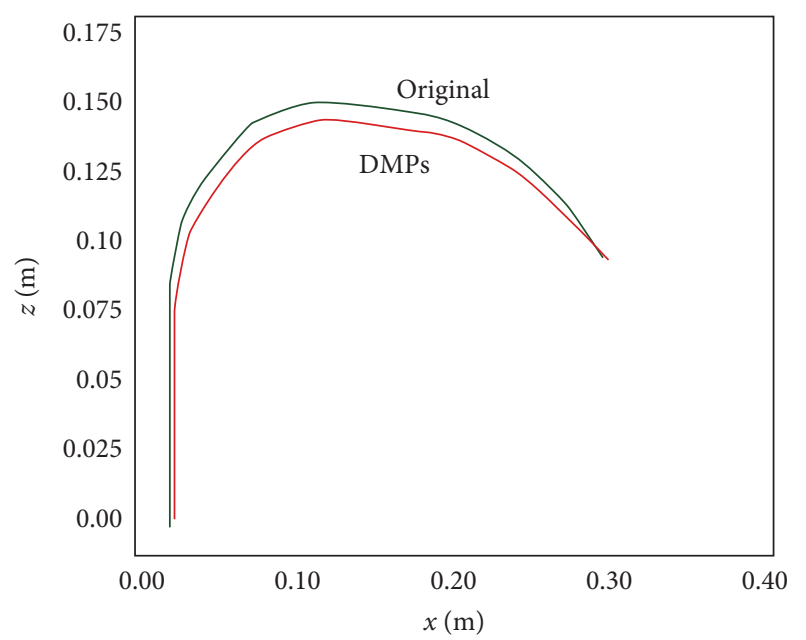

Figure 4: Curves of DMPs modulated according to the original target curve.

TABLE 1: Running time of DMPs in different Gaussian kernels.

\begin{tabular}{lc}
\hline Gaussian kernel & Running time (s) \\
\hline 10 & 0.122 \\
50 & 0.133 \\
100 & 0.173 \\
\hline
\end{tabular}

of appropriate Gaussian kernel functions needs to be considered when the actual embedded Internet of Health Things system is running.

The DMP planning will be discussed below, where the number of Gaussian kernel functions is set to 50 , and the number of weights is 50 . Since the dimension is 2 , the total number of weights is 100 . The end point of the target curve is $(0.3,0.1)$. It should be noted that the more cores, the higher the fitting accuracy. However, the calculation speed will decrease. The end point of the target curve is $n$. It should be noted that the more cores, the higher the fitting accuracy. However, the calculation speed will decrease. The first step is selected as the target curve, and the curve after DMP fitting is shown in Figure 4 . The horizontal axis represents the position of the ankle joint from the origin in the horizontal direction, and the vertical axis represents the distance of the ankle joint from the origin in the $Z$ direction.

The figure above shows that DMPs can learn the original curve very well. By setting multiple targets, dynamic modulation is performed, and $h=0.1 \mathrm{~m}$ is the original curve. The target positions $(0.34,0.19)$ ,$(0.27,0.15)$, and $(0.3,0.12)$ are, respectively, set. The curve after DMP modulation is shown in Figure 5. The horizontal axis represents the position of the ankle joint from the origin in the horizontal direction, and the vertical axis represents the distance of the ankle joint from the origin in the $Z$ direction.

As can be seen from Figure 5, it can be seen that the DMP method can perform dynamic modulation according to the transformation of the target point but cannot adjust the curve pattern according to the target point. Therefore, it can also be explained that the multiscale gait can be modulated by setting the distance and height from the steps.

In the same scene, we collect normal people's gait data at different scales up and down or artificially use some tools to generate curves. We set the data set to $\left(h^{d}, y_{i}^{d}\right), d$ represents curves of different heights, and the corresponding height of the curve is $h$. Among them, $i$ is the $i$-th point in the $d$-th curve. The corresponding virtual force in the corresponding DMPs is calculated by the following formula:

$$
f_{i}^{d}=y_{i}^{\prime \prime}-\alpha_{y}\left(\beta_{y}\left(g-y_{i}^{d}\right)-y_{i}^{\prime d}\right) .
$$

The curve in the above formula is obtained by collecting. By setting a time or taking the acquisition time $t$ as the standard, the points collected by the curve are differentiated to obtain the speed, and the second-order difference is used to obtain the corresponding acceleration. $\alpha_{y}$ and $\beta_{y}$ are constant terms like the parameters in DMPs. Then, after calculating the force required for the forcing term in DMPs, it is necessary to use the partial weighted regression method to obtain the corresponding weight. The specific method used is the same as that in the basic DMPs. In this paper, the least square method is used to establish the mapping relationship between step height and weight. The least square method has the ability to quickly fit the curve, especially the matrix form can be conveniently and quickly calculated, which also provides a good foundation for the application in the lower limb exoskeleton controller and reduces the demand for capacity.

The basic principle of the least squares method is to establish the functional relationship between related variables and corresponding values. For example, if the collected point set is $\left(x_{i}, y_{i}\right), i=0,1,2, \ldots, n$, the functional relationship between $x_{i}$ and $y_{i}$ needs to be established. Under normal circumstances, this kind of functional relationship is impossible to obtain directly. At this time, it is necessary to adopt an approximate method to establish the functional 


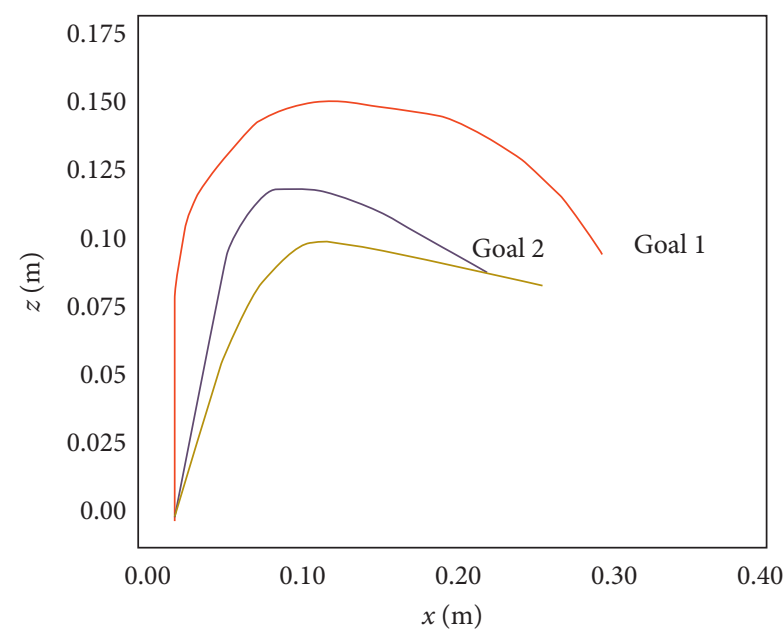

Figure 5: Curves of DMPs modulated according to different target points.

relationship. General functions can be expanded into $n$ order polynomials, so the fitting function here is selected as a higher-order polynomial, and the function relationship is defined as follows:

$$
g(x)=a_{0}+a_{1} x+\cdots+a_{n-1} x^{n-1} .
$$

In the above formula, $a$ is a specific parameter, and $x$ is a variable. The problem at this time turns into the problem of how to obtain the parameters of the higher-order polynomial. We can define a loss function and obtain the minimum parameters of the whole curve and the target curve by solving the minimum value of the loss function as ideal parameters to achieve the purpose of fitting. The loss function is defined as

$$
J(a)=\min \sum\left(g\left(x_{i}\right)-y_{i}\right)^{2} .
$$

Through optimization to minimize the overall mean square error, the optimal parameters can be obtained. In addition to the iterative method, there is also a matrix method to solve the optimal parameters. The above formula is rewritten into matrix form:

$$
\left\{\begin{array}{l}
g(x)=A X, \\
A=\left[a_{0}, a_{1}, \ldots, a_{n-1}\right], \\
X=\left[1, x, \ldots, x^{n-1}\right] .
\end{array}\right.
$$

Among them, $X$ is a matrix composed of variables, and $A$ is a parameter matrix. The loss function is

$$
J(A)=\min \left(\frac{1}{2}(A X-G)^{T}(A X-G)\right) .
$$

Among them, $Y$ is the value matrix corresponding to $X$. When deriving the derivation of the parameters of the above loss function, in order to minimize the overall value, the extreme point of $J(A)$ needs to be selected, and the first derivative of the loss function is 0 . Through transformation, the following results can be obtained:

$$
A=\left(X^{T} X\right)^{-1} X^{T} G
$$

When the least squares method fits the weights of DMPs, $h^{d}$ is the height of the $d$-th curve. First, we give the curve $\left(h^{d}, y_{i}^{d}\right)$, calculate the virtual force $f_{i}^{d}$ of each curve separately, and calculate the virtual force weight matrix $w_{\mathrm{BFs}}^{d}$ of each curve separately. Among them, $d$ is the $d$-th curve, and $\mathrm{BFs}$ is the number of kernel functions. At this time, $\left(h^{d}, w_{\mathrm{BFs}}^{d}\right)$ is as follows:

$$
\left\{\begin{array}{c}
h^{1} \longrightarrow\left[w_{0}^{1}, w_{1}^{1}, \ldots, w_{\mathrm{BFs}}^{1}\right] \\
h^{2} \longrightarrow\left[w_{0}^{2}, w_{1}^{2}, \ldots, w_{\mathrm{BFs}}^{2}\right] \\
\vdots \\
h^{d} \longrightarrow\left[w_{0}^{d}, w_{1}^{d}, \ldots, w_{\mathrm{BFs}}^{d}\right]
\end{array}\right.
$$

The relationship between weight and height established by the least square method is shown in the following formula:

$$
w_{i}=g_{i}(h), \quad i=1,2, \ldots, \text { BFs. }
$$

If it is assumed that the order of the fitting function is $m$, then there are $m$ parameters. If each weight corresponds to $m$ fitting parameters, then there is a fitting parameter of $m *$ BFs size, namely, $A_{m * \mathrm{BFs}}$. Later, using the finally obtained relationship $g(h)$ between the fitting parameters and the height of the step, the weight matrix generated by the height of the step can be obtained by the above formula. By substituting the weight matrix, the output of DMPs can be obtained. The weight of DMPs is transformed into the relationship between the height of the step and the weight of the fitting function. In this way, the height of the steps can directly participate in the adjustment of the curve style, and the method of directly adjusting the weight of DMPs through the height of the steps is realized. The block diagram is shown in Figure 6.

After that, we adopt multiple reference curves and directly introduce the height of the steps into the generation of the gait curve, which further ensured the rationality of the curve, and use the method of dynamic motion primitives to have good scalability. It has better environmental adaptability than the single-curve adjustment method and can avoid the robot from generating an unreasonable gait curve during the movement process, thereby ensuring safer walking of the lower limb exoskeleton.

In the specific use, the standard Internet of Health Things system needs to be attenuated from 1 to 0 , so that the entire Internet of Health Things system can finally converge to the target. This method is generally suitable for offline tasks. In this paper, DMP is required to be able to adapt to online tasks and dynamically adjust the state of the Internet of Health Things system according to changes in foot pressure. Therefore, some changes need to be made to the rules of DMP itself. As shown in Figure 7, the horizontal axis represents time, and the vertical axis represents the control period of the DMPs in the DMPs standard Internet of Health Things system. Through this, we can control the gait time of 


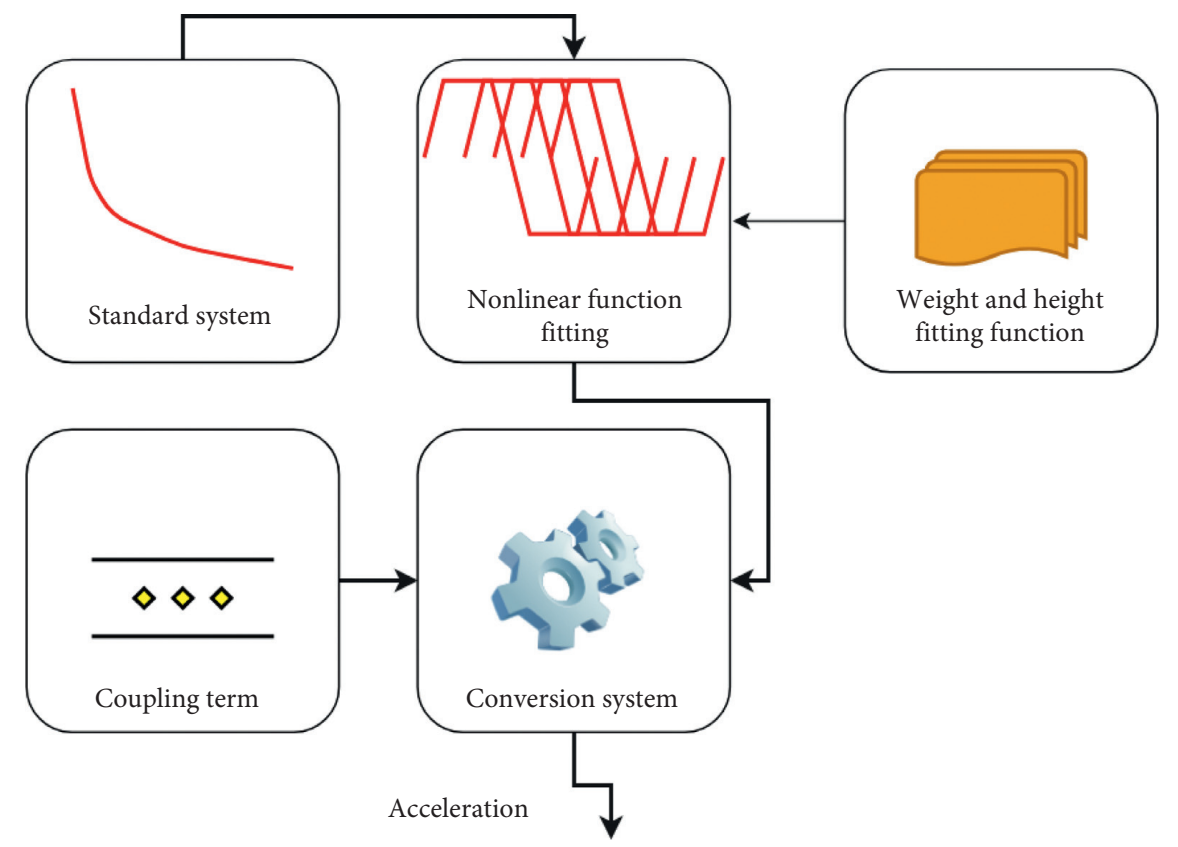

Figure 6: Block diagram of the DMP Internet of Health Things system based on step height.

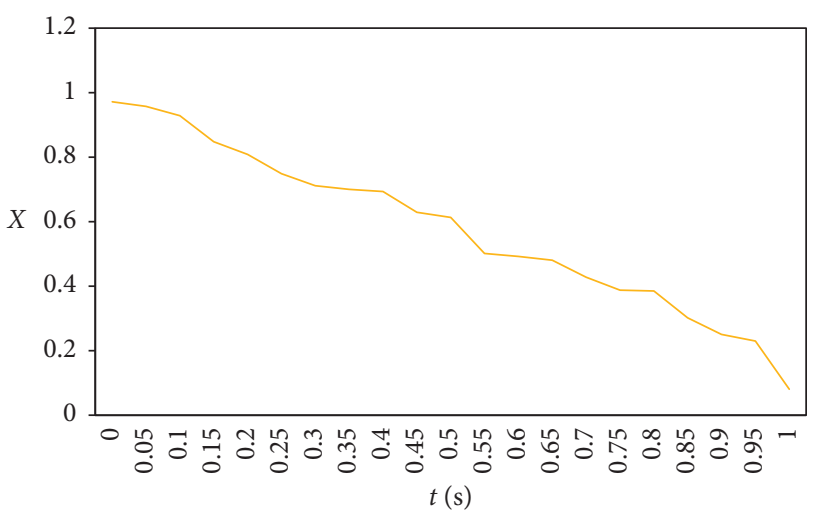

Figure 7: The $x$ of the DMP standard Internet of Health Things system attenuates with $t$.

DMPs; that is, we assign a time scale to $x$ and control the movement during this period.

The overall stepping process of sports rehabilitation is similar to the gait movement process of lower extremity exoskeleton. It can be roughly divided into the following processes: preparation when standing, taking the first step, and then reaching the steps. As shown in Figure 8, the black dots on the soles of the feet represent the center of pressure on the soles of the feet, and the black dots on the human body are the positions of the center of gravity of the person.

Not all processes of the lower limb exoskeleton gait require the participation of the foot pressure interaction item, but only when the forefoot steps on the steps, the process of shifting the center of gravity requires force interaction, which is used to control the movement of the center of foot pressure to the desired position. Then, the DMP relationship with pressure coupling term can be defined as follows:

$$
y^{\prime \prime}=C_{1}\left(\alpha_{y}\left(\beta_{y}(g-y)-y^{\prime}\right)+f\right)+C_{2} A\left(x_{c}(t)-x_{s}\right) .
$$

Among them, $C_{1}$ and $C_{2}$ are used to control whether the basic DMP items and pressure interaction items play a major role. The reason is that in the actual exercise rehabilitation of lower extremity exoskeleton, it is not necessary for the two items to always work together. Here, a pressure detection unit will be introduced to control the two items, which is used to control whose role is stronger. $x_{c}$ and $x_{s}$ are, respectively, the position of the pressure center point of the detected stepping leg and the position of the set pressure center point. The pressure interaction term in this paper only needs to be effective when stepping on a step, and the sole pressure is defined as $P$. The control method of $C_{1}$ and $C_{2}$ in the pressure detection process is defined as follows:

$$
\left\{\begin{array}{lll}
C_{1}=1, & C_{2}=0, & p=0 \\
C_{1}=1, & C_{2}=0, & p>0 .
\end{array}\right.
$$

Among them, $A\left(x_{c}(t)-x_{s}\right)$ is an interactive item of acceleration level, which is mapped to the acceleration that DMP can respond to according to the position of the pressure center of the foot. A single pressure point on the sole of the foot is $f_{i}$. There are $n$ pressure points in total. If the coordinate of each pressure point is $\left(x_{i}, y_{i}\right)$, then the coordinate of the corresponding pressure center point is

$$
\begin{aligned}
& x_{c}=\frac{\sum_{i=1}^{n} f_{i} * x_{i}}{\sum_{i=1}^{n} f_{i}}, \\
& y_{c}=\frac{\sum_{i=1}^{n} f_{i} * y_{i}}{\sum_{i=1}^{n} f} .
\end{aligned}
$$




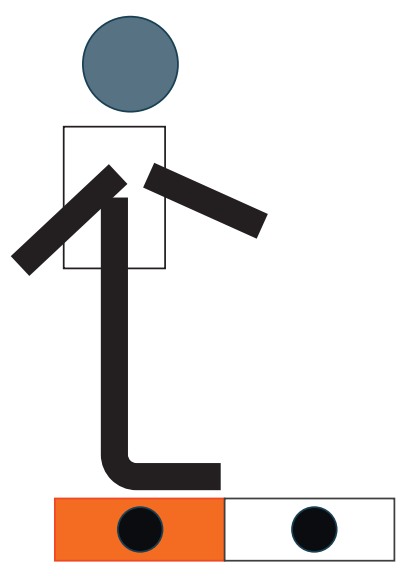

(a)

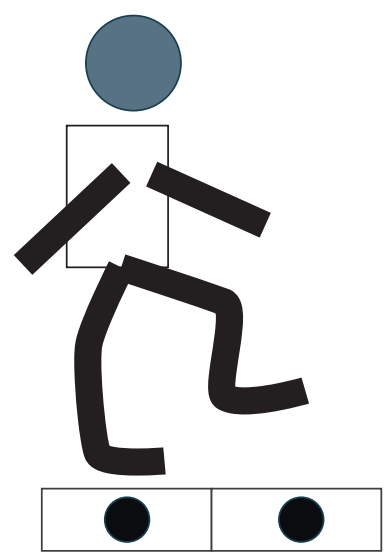

(b)

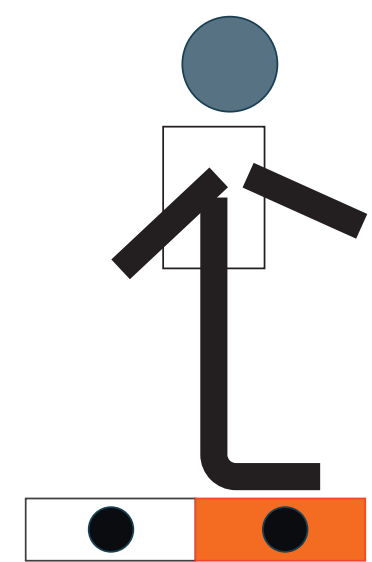

(c)

Figure 8: Gait movement process.

Among them, $x_{c}$ and $y_{c}$ are the calculated pressure centers. It should be noted that here we only need to select the direction of the $x$-axis, which is the direction of foot movement. The coupling term is defined as follows:

$$
\begin{aligned}
A\left(x_{c}(t)-x_{s}\right)= & K\left(x_{c}(t)-x_{s}\right)+D\left(A\left(x_{c}(t)-x_{s}\right)\right. \\
& \left.-A\left(x_{c}(t-1)-x_{s}\right)\right) .
\end{aligned}
$$

Among them, $K$ and $D$ are defined constants, which are used to control the distance from the target and the acceleration level conversion between DMP coupling items. The basic principle is similar to the PD controller. That is, it is used to control itself to reach a desired position and can remain there, until the arrival of the next instruction, and it does not need to be released. The first item is similar to the ratio item in the PD controller to realize the basic adjustment function. The role here is to complete the mapping from the control error to the acceleration level. The second term is similar to the differential term in the PD controller. According to the changing trend, a basic compensation term is added to speed up reaching the target. The overall block diagram is shown in Figure 9, including the standard Internet of Health Things system, nonlinear function fitting items, conversion Internet of Health Things system, and foot pressure coupling items.

After that, it is necessary to verify the effect of different $K$ and $D$ parameters on the Internet of Health Things system. By setting different $K$ and fixing $D$, the sampling period of the Internet of Health Things system is $0.1 \mathrm{~s}$ once. In the actual Internet of Health Things system, the influence of $K$ on the adjustment time of the sole pressure is verified. In the same way, different $D$ and fixed $K$ values are set to verify the influence of different $D$ on the adjustment time. The effect is shown in Figure 10, where Figure 10(a) is the $K$ curve, and Figure $10(\mathrm{~b})$ is the $D$ curve. The horizontal axis represents different $K$ and $D$ values. The vertical axis represents the time for the sampling Internet of Health Things system to adjust to the target point on the sole of the foot, where the position of the target point is set to $0.15 \mathrm{~m}$.

In Figure 10, it can be seen that the value of $k$ plays a major role in the influence of the Internet of Health Things system and can significantly improve the adjustment time. The adjustment of $D$ plays a secondary role, and its adjustment time range is not large, so it mainly plays a supplementary role.

It is verified whether the algorithm in this paper can adjust the desired position of the sole pressure according to the desired target value. In this paper, by setting different pressure center positions of the soles of the feet, the Internet of Health Things system executes the gait movement process and collects the center positions of the sole pressures. The set foot pressure center position is $0.120 \mathrm{~m}, 0.130 \mathrm{~m}, 0.150 \mathrm{~m}$, and $0.170 \mathrm{~m}$, and the result after the Internet of Health Things system is executed is shown in Figure 11. Among them, the hope point represents the expected value, the horizontal axis represents the sampling time, and the vertical axis represents the position of the sole pressure center from the heel:

It can be seen from Figure 11 that the DMP algorithm coupled with the sole pressure can adjust the center position curve of the sole pressure according to the desired position and control it to the vicinity of the desired position.

The following is to verify the effect on the ankle curve of the gait. First, this paper does a basic principle verification on the actual lower extremity exoskeleton Internet of Health Things system. The number of DMP Gaussian kernels is set to 50 , the number of weights is $50, K$ is 3 , and $D$ is 0.01 . In this paper, $K$ and $D$ are derived from the proportional term in the actual debugging and PD controller, which is similar to the coefficient source of the differential term, and the step height is $0.12 \mathrm{~m}$. Figure 12 shows the ankle curve generated by the actual Internet of Health Things system. The horizontal axis represents the distance of the ankle joint from the origin on the $x$-axis, and the vertical axis represents the height of the ankle joint movement.

In Figure 12(b), the basically generated trajectory shows a curve extending in the $Z$ direction at the position of the $x$ axis close to 0.28 point, and the ankle joint curve turns when the height of the ankle joint curve in the $Z$ direction is $0.15 \mathrm{~m}$. However, in Figure 12(a), there is no turning point near $(0.28,0.15)$ to continue downward, which can indicate that the curve planned at the beginning needs to be executed near the target point, which is less than $0.15 \mathrm{~m}$. In Figure 12(b), there is a turning point at a height of $0.15 \mathrm{~m}$. 


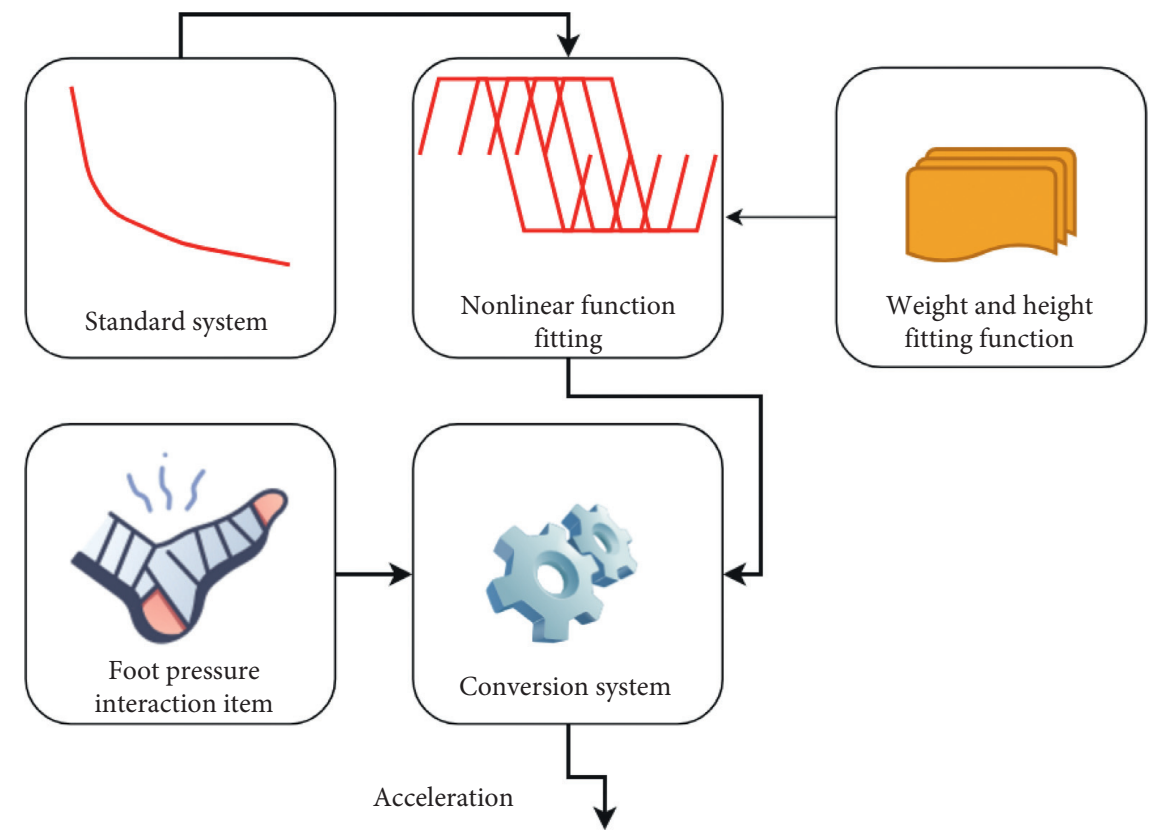

FIGURE 9: Block diagram of foot pressure coupled with dynamic motion primitives.

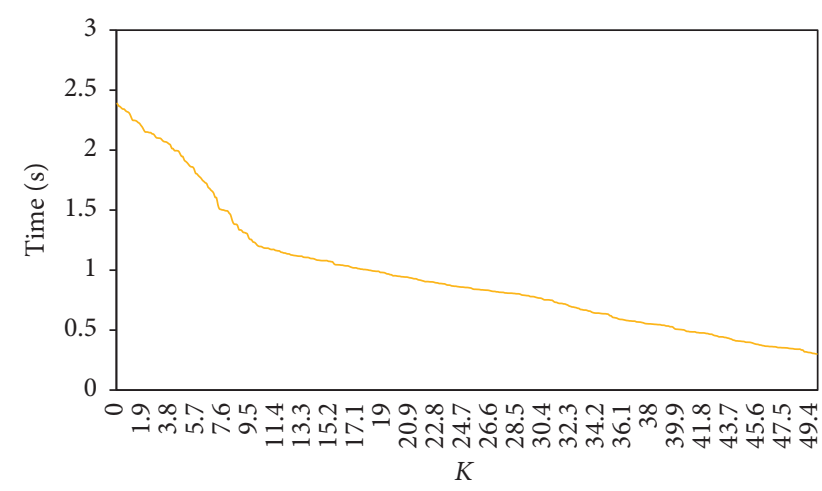

(a)

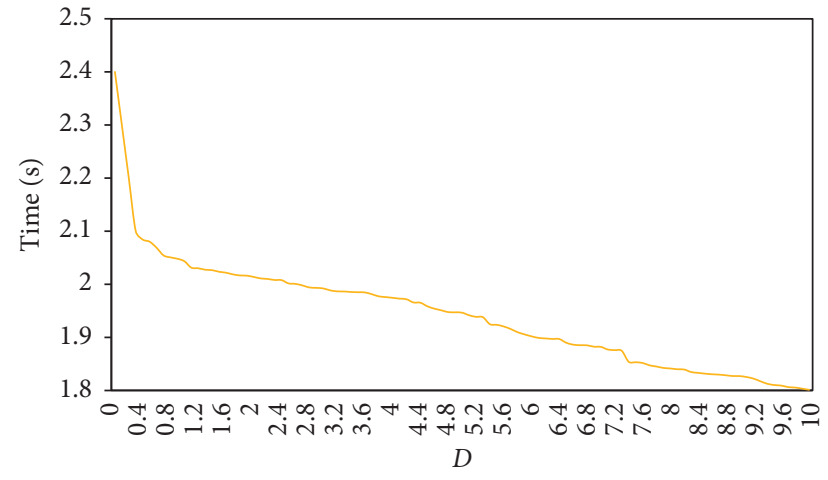

(b)

Figure 10: The influence of $K$ and $D$ parameters on Internet of Health Things system adjustment time. (a) $K$ parameter; (b) $D$ parameter.

After coupling the foot pressure interaction item, when the foot steps up the step, it will touch the step and generate pressure, and then it will actively change the position of the center of gravity indirectly by adjusting the gait. The reason for the modulation in the $Z$ direction is that this type of movement is related to its mechanical structure. Generally, the exoskeleton does not have an active ankle, so the only way to change the center of gravity is by raising the legs. The advantage of this method is that it can quickly respond to the stepping on the steps of the exoskeleton of the lower extremities during the leg step and adjust quickly according to the force feedback. Moreover, it will not have too much influence on the generated curve when there is no pressure on the sole of the foot. Therefore, for the basic gait, it can be guaranteed to be similar to the original trajectory.

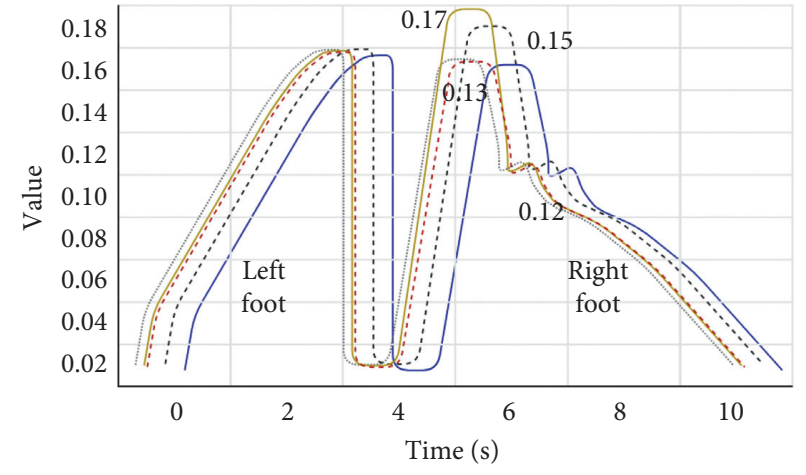

FIGURE 11: Set different desired foot pressure center position after adjusting the foot pressure curve. 


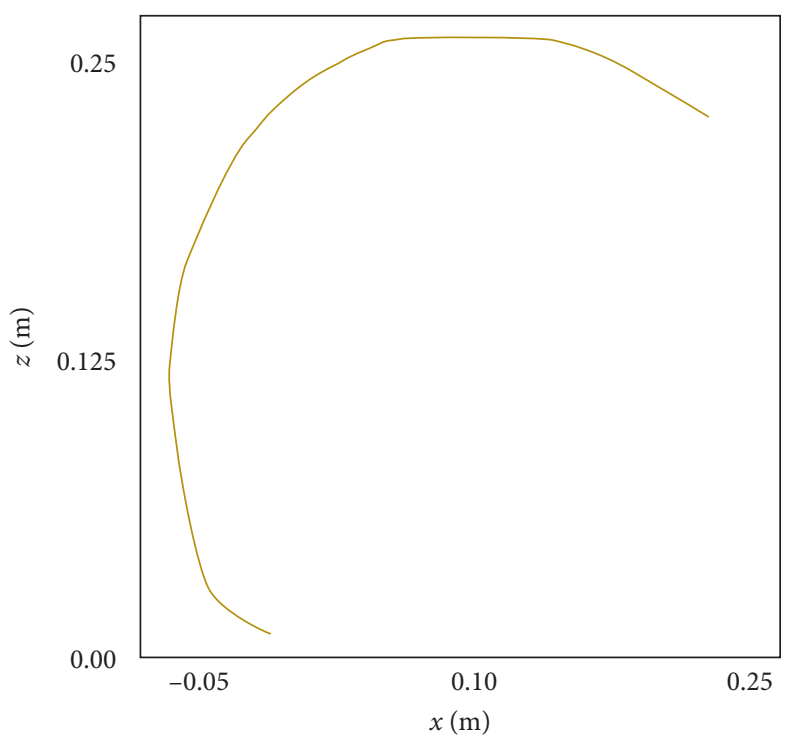

(a)

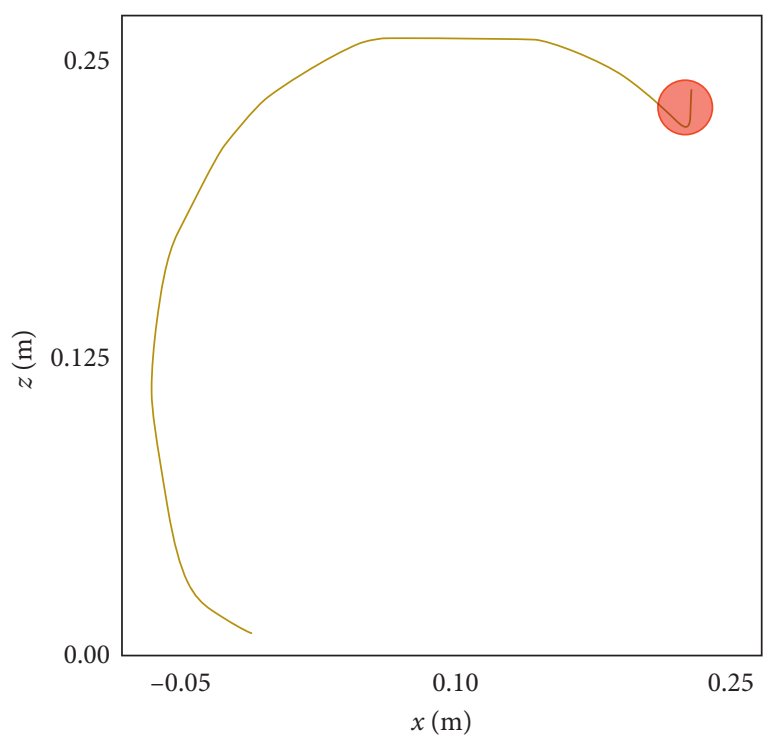

(b)

Figure 12: The first-step ankle joint planning of DMP gait movement. (a) Original plan; (b) foot coupling pressure.

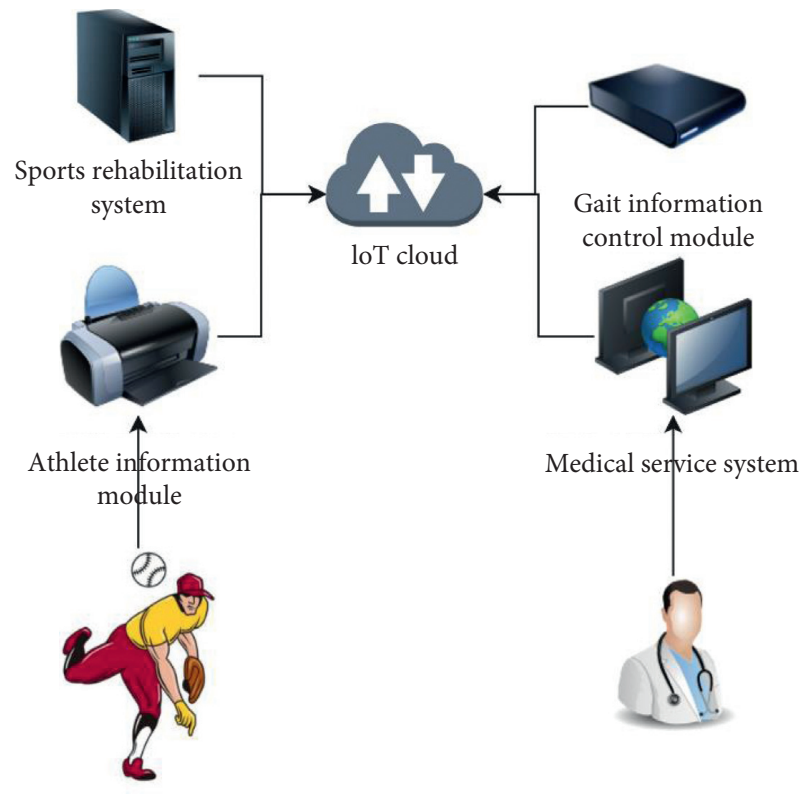

FIGURE 13: System function module.

\section{Rehabilitation Evaluation System Based on Internet of Health Things}

This paper proposes a set of athlete rehabilitation evaluation systems based on the Internet of Health Things and human gait analysis algorithm. This system consists of five main parts: (a) rehabilitation training system; (b) physiological information collection module; (c) patient control terminal; (d) network communication unit; (e) physician server. The system function module is shown as in Figure 13.

The athlete control terminal is mainly composed of a microprocessor-centric embedded platform with a network interface based on the embedded Linux operating system.
The main functions are robot control unit, local communication unit (Bluetooth), game unit, video and audio unit, network interface unit, human-computer interaction unit, etc. The video and audio unit includes the drive of the camera, image acquisition, storage, and display. The network interface unit is responsible for the drive and data transmission of the network interface device. The human-computer interaction unit is responsible for the drive and display of the display screen and touch screen. It is worth noting the network interface unit at the athlete's control end. Because this system emphasizes the Internet of Health Things system, the more important point in the Internet of Health Things system is the powerful network communication capability, as shown in Figure 14.

The physician server needs to provide database support and network interface, open WEB service interface, and support video surveillance. Moreover, the physician server in this system is designed as a cloud service to establish electronic medical records for each user and provide electronic query functions. No matter which terminal (rehabilitation training robot) the user exercises on, as long as the identity recognition is valid, the last exercise setting or customized plan can be continued, as shown in Figure 15.

\section{Model Test}

After constructing the above model, we verify the performance of the model. The model constructed in this paper is an athlete rehabilitation evaluation system based on the Internet of Health Things and human gait analysis algorithms. Therefore, the model performance test and the model practical effect test are carried out during the model performance test. First of all, this paper conducts model performance testing, obtains multiple sets of sports videos through the network, recognizes these videos through the system, and counts the speed and system stability of the system when processing 90 sets of video 


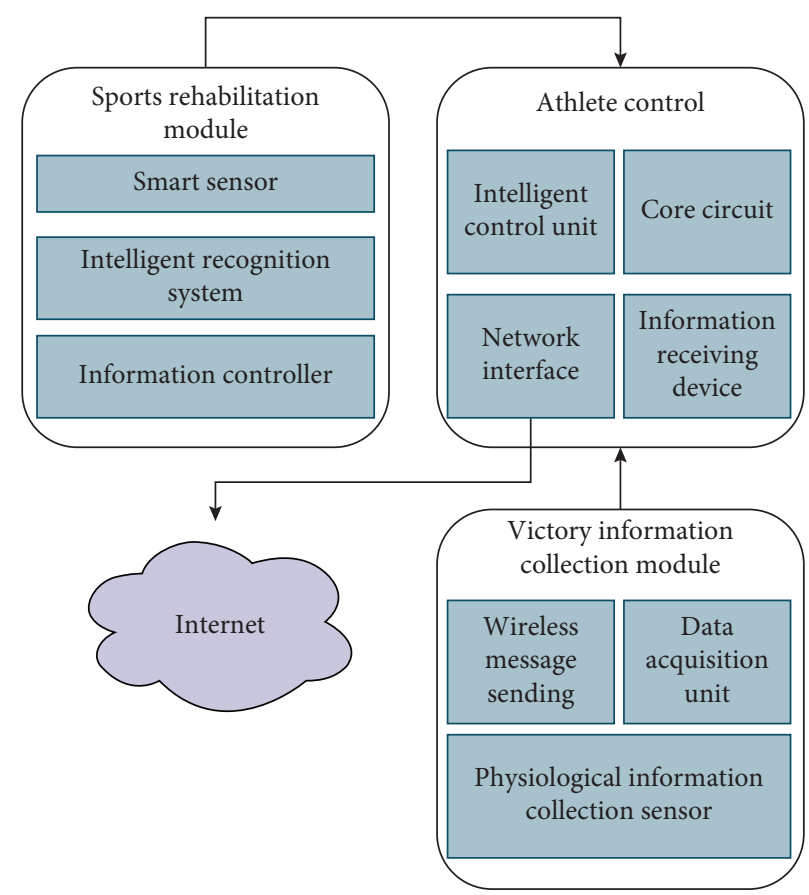

FIGURE 14: The structure diagram of local services in the rehabilitation training system.

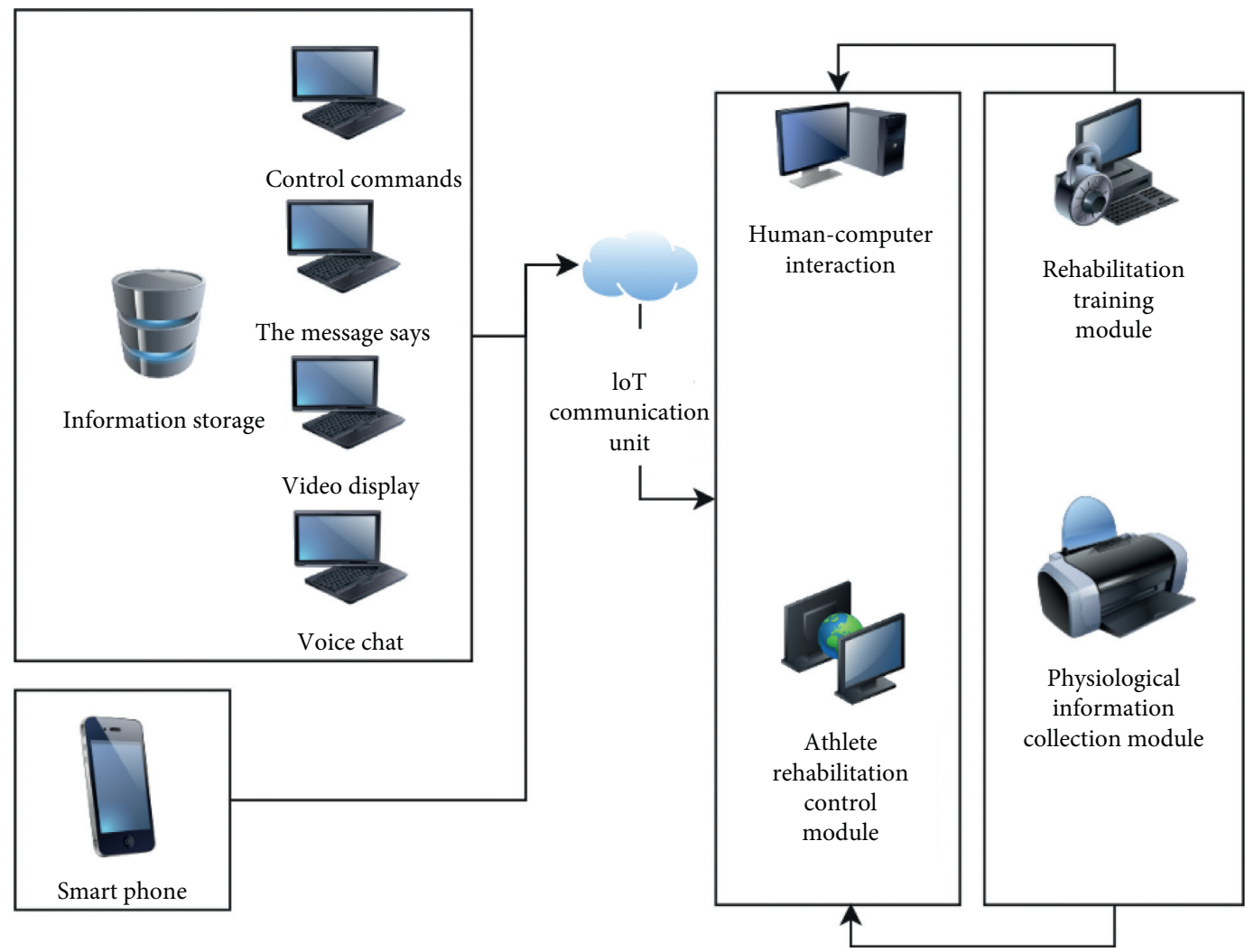

FIGURE 15: Functional block diagram of the remote interactive system. 
TABLE 2: Statistical table of system performance test results.

\begin{tabular}{|c|c|}
\hline No. & Speed (ms) \\
\hline 1 & 138.07 \\
\hline 2 & 101.56 \\
\hline 3 & 147.97 \\
\hline 4 & 96.05 \\
\hline 5 & 116.11 \\
\hline 6 & 109.76 \\
\hline 7 & 137.47 \\
\hline 8 & 117.37 \\
\hline 9 & 103.03 \\
\hline 10 & 107.32 \\
\hline 11 & 103.61 \\
\hline 12 & 126.22 \\
\hline 13 & 119.10 \\
\hline 14 & 133.18 \\
\hline 15 & 106.95 \\
\hline 16 & 121.72 \\
\hline 17 & 106.41 \\
\hline 18 & 109.89 \\
\hline 19 & 101.56 \\
\hline 20 & 132.44 \\
\hline 21 & 134.32 \\
\hline 22 & 111.54 \\
\hline 23 & 119.29 \\
\hline 24 & 118.38 \\
\hline 25 & 129.20 \\
\hline 26 & 114.65 \\
\hline 27 & 105.51 \\
\hline 28 & 149.38 \\
\hline 29 & 124.05 \\
\hline 30 & 105.53 \\
\hline 31 & 144.25 \\
\hline 32 & 114.81 \\
\hline 33 & 118.08 \\
\hline 34 & 110.85 \\
\hline 35 & 108.44 \\
\hline 36 & 107.23 \\
\hline 37 & 129.84 \\
\hline 38 & 123.34 \\
\hline 39 & 129.82 \\
\hline 40 & 115.94 \\
\hline 41 & 97.10 \\
\hline 42 & 141.14 \\
\hline 43 & 118.70 \\
\hline 44 & 141.92 \\
\hline 45 & 136.49 \\
\hline 46 & 115.54 \\
\hline 47 & 105.56 \\
\hline 48 & 122.94 \\
\hline 49 & 125.13 \\
\hline 50 & 124.69 \\
\hline 51 & 113.10 \\
\hline 52 & 129.60 \\
\hline 53 & 119.14 \\
\hline 54 & 145.56 \\
\hline 55 & 133.10 \\
\hline 56 & 146.53 \\
\hline 57 & 121.47 \\
\hline 58 & 117.79 \\
\hline 59 & 147.69 \\
\hline 60 & 147.50 \\
\hline
\end{tabular}

TABle 2: Continued.

\begin{tabular}{lc}
\hline No. & Speed $(\mathrm{ms})$ \\
\hline 61 & 149.74 \\
62 & 119.19 \\
63 & 135.48 \\
64 & 100.70 \\
65 & 109.16 \\
66 & 145.16 \\
67 & 137.74 \\
68 & 106.36 \\
69 & 99.56 \\
70 & 131.96 \\
71 & 148.09 \\
72 & 144.08 \\
73 & 95.82 \\
74 & 141.23 \\
75 & 140.95 \\
76 & 123.96 \\
77 & 126.83 \\
78 & 106.51 \\
79 & 111.39 \\
80 & 135.46 \\
81 & 144.03 \\
82 & 117.19 \\
83 & 134.27 \\
84 & 113.88 \\
85 & 110.19 \\
86 & 148.62 \\
87 & 134.89 \\
88 & 101.88 \\
89 & 126.31 \\
90 & 123.55 \\
\hline & \\
\hline 0 &
\end{tabular}

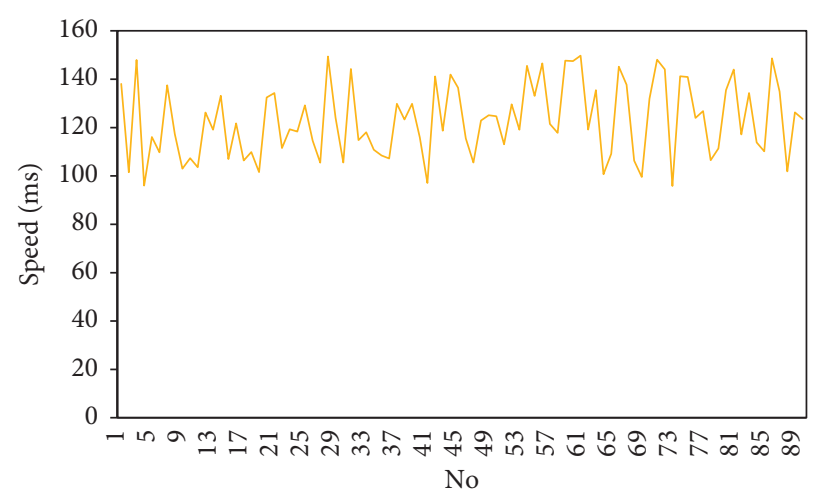

FIGURE 16: Statistical diagram of system performance test results.

TABLE 3: Statistical table of system practice effect.

\begin{tabular}{lcc}
\hline No. & Loss assessment & Rehabilitation effect \\
\hline 1 & 88.71 & 71.29 \\
2 & 83.89 & 81.58 \\
3 & 81.73 & 74.02 \\
4 & 84.37 & 84.93 \\
5 & 80.69 & 82.02 \\
6 & 90.42 & 78.81 \\
7 & 83.90 & 81.90 \\
\hline
\end{tabular}


TABle 3: Continued.

\begin{tabular}{|c|c|c|}
\hline No. & Loss assessment & Rehabilitation effect \\
\hline 8 & 89.10 & 84.25 \\
\hline 9 & 79.18 & 79.27 \\
\hline 10 & 89.17 & 78.54 \\
\hline 11 & 82.97 & 70.84 \\
\hline 12 & 88.76 & 71.36 \\
\hline 13 & 81.66 & 82.32 \\
\hline 14 & 85.52 & 73.68 \\
\hline 15 & 88.72 & 77.25 \\
\hline 16 & 81.70 & 74.10 \\
\hline 17 & 83.81 & 82.46 \\
\hline 18 & 80.13 & 83.21 \\
\hline 19 & 82.01 & 80.19 \\
\hline 20 & 81.72 & 84.19 \\
\hline 21 & 84.02 & 72.66 \\
\hline 22 & 81.60 & 81.13 \\
\hline 23 & 90.12 & 83.67 \\
\hline 24 & 87.44 & 73.05 \\
\hline 25 & 81.47 & 85.10 \\
\hline 26 & 85.04 & 84.65 \\
\hline 27 & 80.35 & 71.05 \\
\hline 28 & 88.92 & 71.57 \\
\hline 29 & 79.23 & 72.40 \\
\hline 30 & 81.90 & 84.44 \\
\hline 31 & 88.73 & 75.41 \\
\hline 32 & 83.42 & 85.84 \\
\hline 33 & 87.91 & 77.13 \\
\hline 34 & 90.71 & 85.34 \\
\hline 35 & 79.43 & 78.48 \\
\hline 36 & 88.03 & 85.41 \\
\hline 37 & 79.55 & 72.53 \\
\hline 38 & 82.69 & 85.60 \\
\hline 39 & 80.56 & 81.04 \\
\hline 40 & 82.41 & 85.18 \\
\hline 41 & 84.92 & 79.39 \\
\hline 42 & 89.00 & 74.38 \\
\hline 43 & 90.45 & 85.42 \\
\hline 44 & 83.28 & 71.98 \\
\hline 45 & 85.81 & 80.77 \\
\hline 46 & 82.80 & 80.06 \\
\hline 47 & 85.81 & 73.93 \\
\hline 48 & 89.15 & 80.74 \\
\hline 49 & 83.05 & 79.18 \\
\hline 50 & 85.26 & 83.05 \\
\hline 51 & 84.30 & 83.70 \\
\hline 52 & 81.58 & 79.31 \\
\hline 53 & 84.07 & 77.78 \\
\hline 54 & 82.21 & 81.09 \\
\hline 55 & 79.93 & 85.17 \\
\hline 56 & 85.81 & 80.83 \\
\hline 57 & 86.62 & 78.15 \\
\hline 58 & 89.75 & 77.54 \\
\hline 59 & 81.02 & 71.31 \\
\hline 60 & 83.88 & 75.40 \\
\hline 61 & 83.90 & 75.91 \\
\hline 62 & 88.79 & 84.82 \\
\hline 63 & 82.80 & 84.16 \\
\hline 64 & 85.12 & 74.87 \\
\hline 65 & 81.56 & 70.76 \\
\hline 66 & 79.65 & 83.47 \\
\hline 67 & 87.37 & 80.83 \\
\hline 68 & 80.34 & 82.36 \\
\hline
\end{tabular}

TABle 3: Continued.

\begin{tabular}{|c|c|c|}
\hline No. & Loss assessment & Rehabilitation effect \\
\hline 69 & 90.77 & 71.23 \\
\hline 70 & 85.55 & 79.62 \\
\hline 71 & 87.90 & 70.71 \\
\hline 72 & 89.76 & 75.76 \\
\hline 73 & 82.04 & 83.21 \\
\hline 74 & 89.12 & 69.79 \\
\hline 75 & 85.85 & 75.70 \\
\hline 76 & 80.20 & 71.59 \\
\hline 77 & 82.97 & 82.37 \\
\hline 78 & 79.31 & 71.32 \\
\hline 79 & 80.91 & 80.43 \\
\hline 80 & 79.04 & 83.90 \\
\hline 81 & 81.99 & 75.09 \\
\hline 82 & 80.03 & 75.52 \\
\hline 83 & 88.32 & 69.79 \\
\hline 84 & 80.86 & 87.70 \\
\hline 85 & 80.09 & 72.27 \\
\hline 86 & 79.94 & 70.92 \\
\hline 87 & 90.52 & 75.49 \\
\hline 88 & 84.69 & 87.88 \\
\hline 89 & 88.64 & 75.71 \\
\hline 90 & 84.87 & 83.35 \\
\hline 91 & 88.64 & 76.19 \\
\hline 92 & 84.14 & 70.97 \\
\hline 93 & 86.52 & 82.10 \\
\hline 94 & 84.87 & 85.96 \\
\hline 95 & 86.23 & 87.22 \\
\hline 96 & 84.48 & 87.76 \\
\hline 97 & 87.23 & 87.29 \\
\hline 98 & 81.79 & 70.17 \\
\hline 99 & 84.57 & 80.27 \\
\hline 100 & 79.57 & 78.73 \\
\hline
\end{tabular}

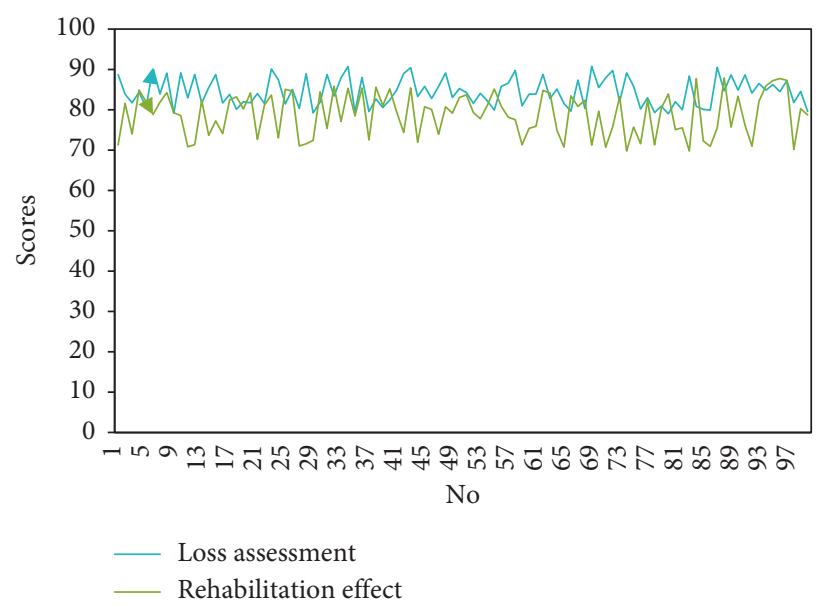

Figure 17: Statistical diagram of system practice effect.

information. The results are shown in Table 2 and Figure 16.

Judging from the test results in Table 2 and Figure 16, it can be seen that the IoT system constructed in this paper has good stability and gives full play to the performance superiority of the IoT system. Next, we will study the 
effects of athletes' rehabilitation assessment in this paper. The sports injury assessment and rehabilitation effect scores of 100 college physical education students are used as the test standards. The results are shown in Table 3 and Figure 17.

From the above practical results, it can be seen that the practice effect of the athlete rehabilitation evaluation system constructed in this paper is very good, which is in line with the expected goal of constructing the system in this paper.

\section{Conclusion}

This paper studies and designs an athlete rehabilitation evaluation system based on the Internet of Health Things and human gait analysis algorithms and designs and completes the overall system plan, measurement and control system, and human-computer interaction. Moreover, this paper proposes a multiscale stair gait planning algorithm based on dynamic motion primitives. In the scene of exoskeleton going up and down stairs, DMPs relying on a single-curve learning method will cause the dynamic motion primitives to appear imbalance of scaling when it is modulated according to different target points. Since such gait may cause safety hazards, this paper optimizes DMPs. Based on multiple curves of the same type and different heights, a mapping relationship between step height and weight is established, and the height of the stairs can directly affect the gait style, which avoids the unbalance of curve scaling. Moreover, this paper constructs the overall structural framework of the system model according to actual needs and makes full use of the advantages of the Internet of Health Things system to improve system performance. In addition, this paper designs experiments to study the performance of the system data processing and the effect of the system on athletes' rehabilitation evaluation and rehabilitation guidance. Through experimental research, the human gait recognition algorithm constructed in this paper has good effects and can play an important role in sports rehabilitation of athletes. At the same time, the system constructed in this paper has certain advantages over traditional sports rehabilitation systems with the support of algorithms. In subsequent practice, the system built in this article can be used to expand.

\section{Data Availability}

The raw/processed data required to reproduce these findings cannot be shared at this time as the data also form part of an ongoing study.

\section{Conflicts of Interest}

The authors declare that there are no conflicts of interest regarding the publication of this paper.

\section{References}

[1] L. G. Appelbaum and G. Erickson, "Sports vision training: a review of the state-of-the-art in digital training techniques,"
International Review of Sport and Exercise Psychology, vol. 11, no. 1, pp. 160-189, 2018.

[2] N. Bennour, "Teaching practices and student action in physical education classes: perspectives for teacher education," Creative Education, vol. 06, no. 10, pp. 934-944, 2015.

[3] M. Bulat, N. Korkmaz Can, Y. Z. Arslan, and W. Herzog, "Musculoskeletal simulation tools for understanding mechanisms of lower-limb sports injuries," Current Sports Medicine Reports, vol. 18, no. 6, pp. 210-216, 2019.

[4] N. Cutforth and E. S. Belansky, "A community-engaged approach to translating research into practice: a physical education story," Progress in Community Health Partnerships: Research, Education, and Action, vol. 9, no. 4, pp. 571-582, 2015.

[5] S. á Fátima, A. Marques, N. B. F. Rocha et al., "Kinematic parameters of throwing performance in patients with schizophrenia using a markerless motion capture system," Somatosensory Research, vol. 32, no. 2, pp. 77-86, 2015.

[6] R. Giannetti, A. Petrella, J. Bach et al., "In vivo bone position measurement using high-frequency ultrasound validated with 3-D optical motion capture systems: a feasibility study," Journal of Medical \& Biological Engineering, vol. 37, no. 7, pp. 1-8, 2017.

[7] P. S. Glazier and S. Mehdizadeh, "Challenging conventional paradigms in applied sports biomechanics research," Sports Medicine, vol. 49, no. 2, pp. 171-176, 2019.

[8] D. Holden, J. Saito, and T. Komura, "A deep learning framework for character motion synthesis and editing," ACM Transactions on Graphics, vol. 35, no. 4, pp. 1-11, 2016.

[9] E.-R. Hwang and T.-Y. Kim, "Intensification of the education of public health, hygiene, and martial arts during the Japanese colonial period (1937-1945)," Journal of Exercise Rehabilitation, vol. 14, no. 2, pp. 160-167, 2018.

[10] O. V. Ivashchenko, O. O. Kapkan, and O. O. Kapkan, "Simulation of process of 14-15 years old girls' training of light athletic and gymnastic exercises," Pedagogics, Psychology, Medical-Biological Problems of Physical Training and Sports, vol. 19, no. 8, pp. 32-39, 2015.

[11] M. A. Khan, "Multiresolution coding of motion capture data for real-time multimedia applications," Multimedia Tools \& Applications, vol. 76, no. 15, pp. 1-16, 2016.

[12] O. M. Khudolii, O. V. Ivashchenko, S. S. Iermakov et al., "Computer simulation of junior gymnasts' training process," Science of Gymnastics Journal, vol. 8, no. 3, pp. 215-228, 2016.

[13] M.-K. Kim, T. Y. Kim, and J. Lyou, "Performance improvement of an AHRS for motion capture," Journal of Institute of Control, Robotics and Systems, vol. 21, no. 12, pp. 1167-1172, 2015.

[14] A. Lopatiev, O. Ivashchenko, O. Khudolii et al., "Systemic approach and mathematical modeling in physical education and sports," Journal of Physical Education and Sport (JPES), vol. 17, no. 1, pp. 146-155, 2017.

[15] T. Mullen, J. Highton, and C. Twist, "The internal and external responses to a forward-specific rugby league simulation protocol performed with and without physical contact," International Journal of Sports Physiology and Performance, vol. 10, no. 6, pp. 746-753, 2015.

[16] D. Mulligan, K. R. Lohse, and N. J. Hodges, “An actionincongruent secondary task modulates prediction accuracy in experienced performers: evidence for motor simulation," Psychological Research, vol. 80, no. 4, pp. 496-509, 2016.

[17] D. Murase, K. Yokoyama, K. Fujii, Y. Hasegawa, and Y. Yamamoto, "Baseball catching patterns differ according to 
task constraints," Advances in Physical Education, vol. 6, no. 3, pp. 151-157, 2016.

[18] P. D. Owen and N. King, "Competitive balance measures in sports leagues: the effects of variation in season length," Economic Inquiry, vol. 53, no. 1, pp. 731-744, 2015.

[19] P. B. Shull, W. Jirattigalachote, M. A. Hunt, M. R. Cutkosky, and S. L. Delp, "Quantified self and human movement: a review on the clinical impact of wearable sensing and feedback for gait analysis and intervention," Gait \& Posture, vol. 40, no. 1, pp. 11-19, 2014

[20] E. Cippitelli, S. Gasparrini, S. Spinsante, and E. Gambi, "Kinect as a tool for gait analysis: validation of a real-time joint extraction algorithm working in side view," Sensors, vol. 15, no. 1, pp. 1417-1434, 2015.

[21] M. Basso, M. Galanti, G. Innocenti et al., "Pedestrian dead reckoning based on frequency self-synchronization and body kinematics," IEEE Sensors Journal, vol. 17, no. 2, pp. 534-545, 2016.

[22] A. Ahmadi, F. Destelle, L. Unzueta et al., "3D human gait reconstruction and monitoring using body-worn inertial sensors and kinematic modelling," IEEE Sensors Journal, vol. 16, no. 24, pp. 8823-8831, 2016.

[23] A. Puupponen, T. Wainio, B. Burger, and T. Jantunen, "Head movements in finnish sign language on the basis of motion capture data: a study of the form and function of nods, nodding, head thrusts, and head pulls," Sign Language and Linguistics, vol. 18, no. 1, pp. 41-89, 2015.

[24] K. R. Ridderinkhof and M. Brass, "How kinesthetic motor imagery works: a predictive-processing theory of visualization in sports and motor expertise," Journal of Physiology-Paris, vol. 109, no. 1-3, pp. 53-63, 2015.

[25] D. Sghaier, S. Elandoulsi, M. Mami, and A. Bouassida, "Physical education teacher's training in swimming under the joint didactic action," Creative Education, vol. 6, no. 22, pp. 2433-2437, 2015.

[26] S. Matheus Maligere de and C. L. D. Silva, "Body practices and Brazilian culture: pedagogical contributions to physical education professionals," Procedia Social \& Behavioral Sciences, vol. 174, pp. 434-440, 2015.

[27] K. A. Tivener and D. S. Gloe, "The effect of high-fidelity cardiopulmonary resuscitation (CPR) simulation on athletic training student knowledge, confidence, emotions, and experiences," Athletic Training Education Journal, vol. 10, no. 2, pp. 103-112, 2015.

[28] J. Yang, "The simulation of table tennis during the course of sports," Caribbean Journal of Science, vol. 52, no. 4, pp. 1561-1564, 2019.

[29] A. Zhang, X. K. Yan, and A. G. Liu, "An introduction to a newly-developed "acupuncture needle manipulation trainingevaluation system" based on optical motion capture technique," Acupuncture Research, vol. 41, no. 6, pp. 556-559, 2016.

[30] H. Zhang, L. Wang, S. Chu, S. Chen, H. Meng, and G. Liu, "Application of optical motion capture technology in power safety entitative simulation training system," Optics and Photonics Journal, vol. 6, no. 8, pp. 155-163, 2016.

[31] Z.-M. Zhou and Z.-W. Chen, "A survey of motion capture data earning as high dimensional time series," International Journal of Multimedia and Ubiquitous Engineering, vol. 10, no. 9, pp. 17-30, 2015. 